\section{Gibberellins and Inhibitors in the Rice Plant}

\section{Shin KurogochI, Noboru Murofushi, Yasuo OTA* and Nobutaka TAKAHASHI

\author{
Department of Agricultural Chemistry, \\ The University of Tokyo, Bunkyo-ku, \\ Tokyo, Japan \\ Konosu, Saitama, Japan
} \\ *National Institute of Agricultural Sciences,}

Received October 25, 1977

The occurrence of gibberellin(GA)-like substances in the rice plant (Oryza sativa L.) was shown by Murakami ${ }^{1)}$ for the first time, and later, the presence of endogenous GAs in many cultivars of rice plant has been proved with exceptions in the case of dwarf varieties, "Tan-ginbozu" and "Sankei-10.", Murakami suggested that the major GA in the rice plant was identical with GA in bamboo shoots by thin-layer chromatography(TLC) using various solvent systems and bioassay. ${ }^{3)}$ However, the identification of GAs in rice plants has not been reported yet, because of small content of GAs in vegetative tissues. This paper deals with the identification of GAs in Oryza sativa L. cv. "Nihonbare" (Japonica) and cv. "T-136" (Indica) as well as identification of two growth inhibitors in Japonica rice.

Shoots ( $9 \mathrm{~kg}$, fresh weight) and roots $(3 \mathrm{~kg}$, fresh weight) of Oryza sativa L. cv. "Nihonbare" were collected from paddy field two months after sowing, and ears ( $1 \mathrm{~kg}$, fresh weight) were harvested at full anthesis. Seedlings $(10 \mathrm{~kg}$, fresh weight) were harvested two weeks after sowing into the trays filled with soil. After harvesting plants were homogenized and extracted with methanol twice using approximately 5 liters of solvent per kilogram of tissue. Methanol was removed in vacuo and the aqueous residue was worked up to obtain ethyl acetate-soluble acidic fraction as follows; the aqueous solution was adjusted to $\mathrm{pH} 7$ and was partitioned three times against the half volume of ethyl acetate, the residual aqueous solution being partitioned three times against ethyl acetate after adjustment to $\mathrm{pH} 3$ to give an acidic fraction. The acidic fraction was purified by the following sequence of column chromatographies, (1) charcoal adsorption chromatography, eluted with acetone/water, (2) silicic acid adsorption chromatography, ethyl acetate/hexane, (3) silicic acid partition chromatography, ethyl acetate/hexane, $0.5 \mathrm{~m}$ formic acid as stationary phase, (4) Sephadex LH-20 chromatography, hexane-ethyl acetateacetic acid-methanol-water (100:80:5:40:7, $\mathrm{v} / \mathrm{v}$ ), lower layer being used as stationary phase and upper layer eluting solvent. The active fraction thus obtained was subjected to TLC using Silica gel $\mathrm{GF}_{254}$ (Merck) and ethyl acetate-chloroform-acetic acid (20:8:1, v/v). GAs in the above purification process were monitored by water culture ${ }^{4}$ and micro drop bioassay" using dwarf rice seedlings, "Tanginbozu" and "Waito-C."

The $R f$ value of TLC and the feature of biological activities on dwarf rice seedlings, "Tan-ginbozu" and "Waito-C," of the most purified fraction from the shoots of Japonica variety suggested that the major GA might be $\mathrm{GA}_{19}$. The active fraction was subjected to GC-MS analysis after derivatization to methyl ester trimethylsilyl ether (MeTMS) using diazomethane and pyridine-bis-trimethylsilyl acetamide-trimethylchlorosilane $(1: 2: 1, \mathrm{v} / \mathrm{v})$. The fragment ions at $m / e 462\left(\mathrm{M}^{+}\right), 434,402$ and 374 , which are characteristic of $\mathrm{GA}_{19} \mathrm{MeTMS}$, were observed in the mass spectrum obtained by scanning at the GC peak with the same retention time as that of the authentic specimen. Thus, it was unequivocally confirmed that the major $\mathrm{GA}$ in the rice plant was $\mathrm{GA}_{19}$.

In the same way as in the case of the shoots, the roots and ears of Japonica variety $\mathrm{cv}$. "Nihonbare" were also examined and these tissues were found to contain $\mathrm{GA}_{19}$ as the major GA. The content of $\mathrm{GA}_{19}$ in various tissues of the rice plant was estimated to be ranged $0.5 \sim 1.5 \mu \mathrm{g} \mathrm{GA}_{3}$ equivalent per $\mathrm{kg}$ of tissue. Minor GAs in the rice were detected by bioassay but identification by GC-MS was unsuccessful due to their minute amount. However, pre- 


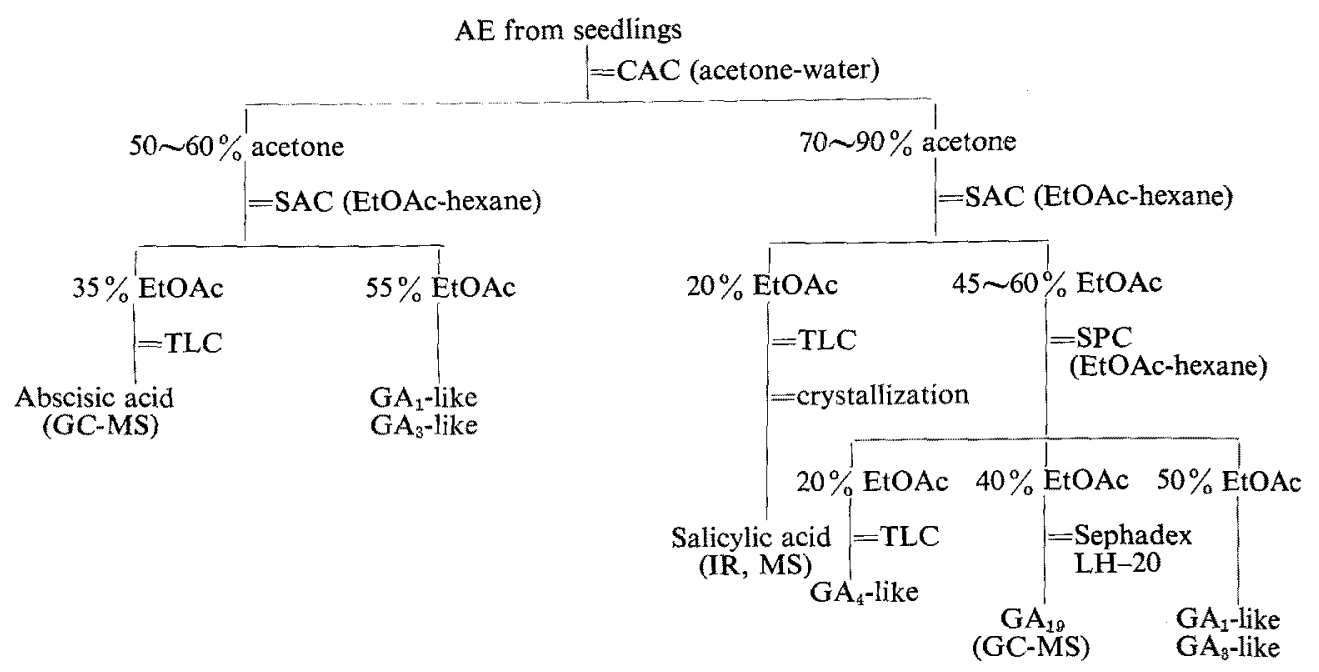

FIG. 1. The Purification Procedure for Acidic Ethyl Acetate-soluble Fraction (AE) from the Seedlings of Oryza sativa L. cv. "Nihonbare."

CAC: charcoal adsorption chromatography, SAC: silicic acid adsorption chromatography, SPC: silicic acid partition chromatography, TLC: silica gel thin-layer chromatography, EtOAc: ethyl acetate.

liminary mass fragmentgraphy analysis suggested that they might be $\mathrm{GA}_{1}, \mathrm{GA}_{3}$ and $\mathrm{GA}_{4}$. Further experiments are under way to identify the minor GAs more firmly.

Two acidic growth inhibitors in the seedlings of Japonica variety were detected by GA bioassay. One was isolated in crystalline state and identified as salicylic acid by the direct comparison of IR spectrum with that of authentic specimen. The content of salicylic acid was $1 \sim 1.5 \mathrm{mg}$ per $\mathrm{kg}$ of fresh weight. The other was identified as abscisic acid by GC-MS. The isolation and identification process of plant regulators from seedlings of the rice plant was summarized in Fig. 1.
$\mathrm{GA}_{19}$ was also found to be the major $\mathrm{GA}$ in seedlings of Indica cv. "T-136." This suggests that both Japonica and Indica variety have the same GA, though they exhibit the different feature of growth pattern.

\section{REFERENCES}

1) Y. Murakami, Bot. Mag. Tokyo, 73, 186 (1960).

2) H. Suge and Y. Murakami, Plant Cell Physiol., 9, 411 (1968).

3) Y. Murakami, Proceedings, of the 7th International Conference of Plant Growth Substances, Canberra, 1970. Springer Verlag, 1972, p. 166.

4) T. Yokota, N. Murofushi, N. Takahashi and M. Katsumi, Phytochemistry, 10, 2943 (1971).

5) Y. Murakami, Bot. Mag. Tokyo, 81, 100 (1968). 